\title{
Avaliação dos níveis plasmáticos do peptídeo natriurético NT-proBNP em cães da raça Poodle em diferentes estágios da doença valvar crônica mitral
}

\author{
[Evaluation of plasma levels of NT-proBNP natriuretic peptide in Poodle dogs at different \\ stages of chronic mitral valve disease] \\ C.G.P. Santana ${ }^{1}$, J.P. Paiva², C.J. Mucha ${ }^{3}$, N.X. Alencar ${ }^{4}$ \\ ${ }^{1}$ Aluno de pós-graduação - Universidade Federal Fluminense - Niterói, RJ \\ ${ }^{2}$ Universidade Federal Rural do Rio de Janeiro - Seropédica, RJ \\ ${ }^{3}$ Prática privada em Cardiologia Veterinária, Buenos Aires, ARG \\ ${ }^{4}$ Universidade Federal Fluminense - Niterói, RJ
}

\begin{abstract}
RESUMO
A doença valvar crônica mitral (DVCM) é comum em cães e pode não causar sintomas clínicos da insuficiência cardíaca (IC) durante anos. O peptídeo natriurético tipo B (BNP) é armazenado nos miócitos ventriculares e secretado para circulação com seu fragmento NT-proBNP, quando ocorre aumento. Este estudo avaliou os níveis plasmáticos do peptídeo natriurético NT-proBNP em cães da raça Poodle em diferentes estágios da DVCM, seguindo as diretrizes do American College of Veterinary Internal Medicine (ACVIM, 2009). Amostras de sangue foram coletadas para determinação do biomarcador NTproBNP para comparação entre grupos. As medianas do NT-proBNP nos grupos estudados foram: $551 \mathrm{pmol} / \mathrm{L}$ (controle), 302pmol/L (grupo B1), 1.033pmol/L (grupo B2), 954pmol/L (grupo C) e $5.541 \mathrm{pmol} / \mathrm{L}$ (grupo D). Mediante o uso de um ponto de corte ideal de $>709 \mathrm{pmol} / \mathrm{L}$, foi possível identificar os cães com aumento cardíaco verdadeiro daqueles sem aumento cardíaco, com sensibilidade de $75 \%$ e especificidade de $100 \%$. O NT-proBNP aumentou de acordo com o avanço dos estágios da DVCM, sendo os estágios B2, C e D aqueles com valores mais elevados desse biomarcador. Para o estágio B2, a mensuração do NT-proBNP mostrou ser uma excelente ferramenta para diagnosticar precocemente o aumento cardíaco em cães da raça Poodle.
\end{abstract}

Palavras-chave: patologia clínica, insuficiência cardíaca, biomarcador

\begin{abstract}
Chronic mitral valve disease (CMVD) is common in dogs, it may not cause clinical symptoms of heart failure (HF) for years. The type B natriuretic peptide (BNP) is stored in the ventricular myocytes and secreted for circulation with its NT-proBNP fragment, when an increase occurs. This study evaluated the plasma levels of the NT-proBNP natriuretic peptide in Poodles at different stages of CMVD, following the guidelines of the American College of Veterinary Internal Medicine (ACVIM, 2009). Blood samples were collected for determination of NT-proBNP biomarker for comparison between groups. This median NTproBNP in the studied groups were: 551pmol/L (Control), 302pmol/L (Group B1), 1,033pmol/L (Group B2), $954 \mathrm{pmol} / \mathrm{L}$ (Group C) and 5,541pmol/L (Group D). Using an ideal cutoff of $>709 \mathrm{pmol} / \mathrm{L}$ it was possible to identify dogs with true heart enlargement of those without a cardiac increase with sensitivity of $75 \%$ and specificity of $100 \%$. NT-proBNP increased according to the progress of the stages of CMVD, being that stages B2, C and D, with the highest values of the biomarker. To stage B2, the NT-proBNP measurement proved to be an excellent tool for early diagnosis of cardiac enlargement in Poodles.
\end{abstract}

Keywords: clinical pathology, heart failure, biomarker

Recebido em 22 de fevereiro de 2017

Aceito em 22 de novembro de 2017

E-mail: cicerocardiovet@hotmail.com 


\section{INTRODUÇÃO}

A doença valvar crônica mitral (DVCM) é o distúrbio cardíaco mais comum (Detweiler; Patterson, 1965), constitui aproximadamente $75 \%$ dos casos vistos na prática veterinária de cães idosos, atinge mais os machos do que as fêmeas e é mais prevalente em cães de porte pequeno (<20kg) (Borgarelli; Haggstrom, 2010). Muitas vezes o sopro cardíaco da insuficiência da mitral e/ou tricúspide é um achado incidental. Os sinais clínicos podem ficar ausentes durante anos e nunca demonstrarem características da IC em alguns cães. Naqueles que exibem os sinais clínicos, estes estão geralmente relacionados à diminuição de exercícios e intolerância a eles; tosse e taquipneia durante esforço físico e episódios de fraqueza transitória ou síncope constituem as queixas comuns entre tutores de cães (Côté et al., 2015).

A definição de biomarcadores diz que são substâncias específicas de um órgão ou tecido em investigação, as quais são sintetizadas e secretadas proporcionalmente à lesão ou doença que o está acometendo (Yonezawa et al., 2010). Os biomarcadores são utilizados com o intuito de auxiliar no diagnóstico clínico de animais com doença cardíaca, com maior acurácia e em menor tempo possível (Pant et al., 2012). Os peptídeos natriuréticos constituem o padrão-ouro para os biomarcadores cardíacos em seres humanos, e o conhecimento sobre sua biologia e sua utilização clínica tem aumentado rapidamente (Gaggin; Januzzi, 2013).

São hormônios natriuréticos estruturalmente semelhantes, bem como suas vias de degradação; em conjunto, regulam o volume e a pressão sanguínea, antagonizam o sistema reninaangiotensina-aldosterona e o sistema nervoso simpático; provocam a broncodilatação e inibem a proliferação de células musculares lisas. Ao antagonizarem o sistema renina-angiotensinaaldosterona e o sistema nervoso simpático, protegem o sistema cardiovascular da sobrecarga de volume (Takemura et al., 2009). A oscilação entre esses sistemas contribui para o desenvolvimento de insuficiência cardíaca congestiva (ICC). Os peptídeos natriuréticos são importantes marcadores de hipertrofia cardíaca e insuficiência cardíaca congestiva (Gaggin; Januzzi, 2013).
O peptídeo natriurético tipo B (BNP) é sintetizado a partir do seu precursor proBNP, que é armazenado nos miócitos ventriculares. $\mathrm{O}$ hormônio BNP ativo é clivado à porção carboxiterminal da molécula precursora e secretado para a circulação com seu respectivo fragmento aminoterminal (NT-proBNP) (MacDonald et al., 2003; Pernobian, 2011). Esse biomarcador cardíaco pode auxiliar na diferenciação de cães com doença cardíaca dos hígidos, além de determinar que animais possuam aumento cardíaco significativo e, ainda, ajudar no diagnóstico diferencial de doenças cardíacas e não cardíacas (Oyama et al., 2009). Sua indicação clínica principal, em se tratanto de pequenos animais, é a deteç̧ão precoce do distúrbio cardíaco em animais assintomáticos, o que ajuda a interceder antes do aparecimento dos sinais clínicos. Ressalta-se que o teste do NTproBNP não substitui a auscultação cardíaca, um método fácil, barato e altamente específico para a doença valvular crônica em cães (Oyama, 2010). Na medicina, ele é utilizado como auxiliar no diagnóstico de pacientes com dispneia ambulatorial e como suporte clínico na suspeita de ICC; além disso, contribui para a avaliação prognóstica e a orientação terapêutica dos pacientes com ICC descompensada (Barretto et al., 2013; Simons, Wauchope, 2015).

No entanto, variação considerável inter-racial na concentração plasmática desses biomarcadores pode ocorrer em cães saudáveis (Sjostrand et al., 2014; Misbach et al., 2013), o que torna fundamental outros estudos para a determinação desses valores em diferentes raças. Considerando que o padrão-ouro para o diagnóstico de cardiopatias, que envolve exames eletrocardiográficos e ecocardiográficos, é relativamente caro e requer experiência e equipamentos (Oyama, 2010), a utilização de biomarcadores pode ser útil na avaliação desses pacientes. O presente estudo teve como objetivo determinar os níveis plasmáticos do peptídeo natriurético NT-proBNP em cães da raça Poodle em diferentes estágios da DVCM.

\section{MATERIAL E MÉTODOS}

Em estudo transversal, foram incluídos 50 cães da raça Poodle, entre machos e fêmeas sem pedigree, de clientes encaminhados ao clínico cardiologista do Hospital Universitário de Medicina Veterinária (Huvet), divididos em 
cinco grupos conforme achados clínicos cardiológicos, seguindo as diretrizes do American College of Veterinary Internal Medicine (ACVIM, 2009) para o diagnóstico e o tratamento da doença valvar crônica em cães. Obesos com escore de condição corporal >5 (sendo entre 3 e 5 o escore ideal), portadores de hemoparasitoses, microfilaremia, doenças infecciosas sistêmicas e sinais clínicos compatíveis com endocrinopatias, foram excluídos deste estudo.

O estudo foi aprovado por um comitê de ética no uso de animais. Todos os responsáveis dos animais que participaram deste estudo foram instruídos a assinarem o termo de consentimento livre e esclarecido, com as descrições adequadas e as informações essenciais ao entendimento da proposta deste estudo. Em cada animal, foi realizado atendimento clínico seguindo semiologia médica com especial atenção ao sistema cardiovascular, de acordo com a metodologia descrita por Fox et al. (1999), com todos os parâmetros anexados ao prontuário médico, contendo histórico, anamnese, exame físico e resultados dos exames complementares.

A aferição da pressão arterial sistêmica não invasiva se fez pelo método oscilométrico de alta definição (Medidor de Pressão Arterial MDpro Beijing Choice Eletronic Tech Co. Ltda.). Realizaram-se três aferições seguidas e, com base na média destas, foi estabelecida a pressão arterial sistólica (PAS), a diastólica (PAD) e a média (PAM). A gravação do registro eletrocardiográfico foi realizada durante dois minutos, empregando-se o aparelho digital 12 Derivações ECGPC $^{\circledR}$ - TEB (Tecnologia Eletrônica Brasileira Ltda.). O ecocardiograma foi realizado com uso do aparelho de ultrassom Sonosite Titan ${ }^{\circledR}$ com transdutor multifrequencial C11/5,0-8,0MHz. A obtenção dos parâmetros ecocardiográficos foi feita conforme descrito por Boon (2011), por meio de ecocardiografia transtorácica, com avaliação qualitativa das quatro câmaras cardíacas, válvulas mitral, aórtica e tricúspide. A partir do modo-M, obteve-se quantitativamente a relação entre o átrio esquerdo e a aorta (AE:Ao), o diâmetro da câmara ventricular esquerda (DVE), a parede livre do ventrículo esquerdo em sístole e diástole (PLVE) e a fração de encurtamento (FEC) miocárdica; com o Doppler contínuo ou pulsátil e em cores, obteve-se gradiente de pressão, velocidades e direções dos fluxos transvalvulares e das vias de saída do coração, visando identificar regurgitação valvar e reconhecer possíveis disfunções sistólicas e diastólicas causadas pela DVCM. A relação AE:Ao obtida pela ecocardiografia foi utilizada como padrãoouro para determinar a existência de remodelamento cardíaco.

As amostras para hemograma foram processadas no Contador Hematológico Automatizado Veterinário Sysmex ${ }^{\circledR}$ - Poch 100 iV; os esfregaços sanguíneos foram confeccionados para contagem diferencial de leucócitos, pesquisa de hemoparasitas e avaliação hematoscópica. A pesquisa de microfilárias ocorreu por meio de duas técnicas: esfregaço sanguíneo e gota espessa (Reagan et al., 2011). Na bioquímica sérica, foram dosados a alanina aminotransferase (ALT), o aspartato aminotransferase (AST), a fosfatase alcalina, as proteínas totais (albumina/globulinas/relação Alb:Glob.), a ureia e a creatinina, seguindo-se as recomendações do fabricante (Labtest Diagnóstica S.A. ${ }^{\circledR}$ ). O método de obtenção da urina foi a cistocentese para realização da urinálise completa (EAS), sendo realizada análise física (avaliação da cor, odor, aspecto e densidade), química (química seca por tiras reagentes para urina - Combur10 Test $\left.^{\circledR} \mathrm{M}\right)$, sedimentoscopia, e a densidade urinária foi determinada em refratômetro portátil (Atago $\left.{ }^{\circledR}\right)$.

Foi utilizado o kit Cardiopet proBNP ${ }^{\circledR}$ canino para ensaio de imunoabsorção enzimática (ELISA) do biomarcador cardíaco NT-proBNP, o qual foi quantificado no Laboratório IDEXX ${ }^{\circledR}$ Ltda. (Califórnia - EUA). As 50 amostras com, no mínimo, 0,5mL de plasma sanguíneo em tubos tipo Eppendorf $\AA$, sem inibidores de proteases, ficaram armazenadas em duplicatas por um período máximo de cinco meses, no freezer, a $-20^{\circ} \mathrm{C}$, até serem encaminhadas ao laboratório IDEXX ${ }^{\circledR}$ (Califórnia - EUA), onde foram processadas.

A análise estatística foi realizada por meio do programa computacional BioEstat 5.3 (Ayres, 2000). Inicialmente foi feita a estatística descritiva para resumir, descrever e compreender os dados obtidos do biomarcador cardíaco NTproBNP de cada grupo separadamente. Foi analisada pelo teste Kolmogorov-Smirnov a normal distribuição das amostras. Em seguida, 
para comparar a concentração plasmática do NTproBNP entre os estágios A, B1, B2, C e D (ACVIM, 2009) causados pela DVCM, utilizouse o teste de Kruskal-Wallis para as amostras que não obtiveram distribuições normais, a fim de indicar se houve diferença significante. Valores de $\mathrm{P}<0,05$ foram considerados significativos. Para avaliar a habilidade e a acurácia do teste diagnóstico do NT-proBNP, utilizou-se a curva ROC (Receiver Operating Characteristic) pelo programa computacional estatístico MEDCALC $^{\circledR}$ (www.medcalc.org). Foi calculada a área sob a curva ROC (AUC) e determinou-se um valor de cut-off ideal, utilizando-se a análise da curva ROC para discriminar os cães sem aumento cardíaco (A, B1) dos cães com aumento cardíaco (B2, C, D). Valores de $\mathrm{P}<0,05$ foram considerados significantes.

\section{RESULTADOS}

Os animais foram atendidos entre o meses de janeiro e junho de 2016 (Huvet). Dos 50 cães utilizados neste estudo, 31 eram fêmeas (62\%) e 19 machos (38\%), distribuídos entre os grupos experimentais independentemente de sexo, idade e estado reprodutivo. Os animais incluídos foram classificados nos cinco grupos de acordo com as diretrizes ACVIM (2009) para diagnóstico, manejo e tratamento da doença valvular crônica (DVC) em cães. A distribuição da população estudada está listada na Tab. 1.

Tabela 1. Número de animais, distribuição de sexo, média de peso e faixa etária entre os grupos

\begin{tabular}{|c|c|c|c|c|c|}
\hline \multicolumn{6}{|c|}{ ACVIM (2009) } \\
\hline Variáveis & A & B1 & B2 & $\mathrm{C}$ & $\mathrm{D}$ \\
\hline Animais (n) & 12 & 10 & 10 & 10 & 8 \\
\hline Sexo & $5 \mathrm{~m} / 7 \mathrm{f}$ & $2 \mathrm{~m} / 8 \mathrm{f}$ & $3 m / 7 f$ & $5 \mathrm{~m} / 5 \mathrm{f}$ & $5 \mathrm{~m} / 3 \mathrm{f}$ \\
\hline Peso (kg) & $\begin{array}{c}5,2^{\mathrm{a}} \\
( \pm 2,5)\end{array}$ & $\begin{array}{l}5,3^{\mathrm{a}} \\
( \pm 2,7)\end{array}$ & $\begin{array}{l}5,6^{\mathrm{a}} \\
( \pm 1,9)\end{array}$ & $\underset{( \pm 1,3)}{5,2^{a}}$ & $\begin{array}{r}5,7^{\mathrm{a}} \\
( \pm 1,9)\end{array}$ \\
\hline Idade (anos) & $\begin{array}{c}5^{\mathrm{a}} \\
( \pm 2,7)\end{array}$ & $\begin{array}{c}9^{\mathrm{ab}} \\
( \pm 1,7)\end{array}$ & $\begin{array}{l}12^{\mathrm{bc}} \\
( \pm 2,4)\end{array}$ & $\begin{array}{c}12^{\mathrm{bc}} \\
( \pm 3,1)\end{array}$ & $\begin{array}{c}15^{\mathrm{c}} \\
( \pm 1,0)\end{array}$ \\
\hline
\end{tabular}

- $\mathrm{n}=$ numero de animais; $\mathrm{kg}=$ quilogramas; $\mathrm{m}=$ machos; $\mathrm{f}=$ fêmeas; $\pm=$ desvio-padrão. Letras diferentes ao longo das ${ }^{-}$ colunas ("a" "b" "c") indicam variáveis que não foram significativas ( $\mathrm{P}>0,05)$.

A mediana da concentração plasmática do NTproBNP do grupo controle (estágio A) foi de 551pmol/L; no grupo do estágio B1, a mediana foi de 302, não apresentando diferença para o grupo controle $(\mathrm{P}>0,05)$. Entretanto, nos grupos dos estágios B2, C e D, a concentração plasmática do NT-proBNP foi diferente $(\mathrm{P}<0,05)$ em comparação ao grupo controle. No grupo do estágio B2, a mediana foi de 1.033pmol/L; no grupo do estágio C, a mediana foi de $954 \mathrm{pmol} / \mathrm{L}$ (primeiro quartil 618,25, terceiro quartil 2.554,75); e a mediana do grupo do estágio D foi de $5.541 \mathrm{pmol} / \mathrm{L}$. Os resultados indicam um aumento da concentração plasmática do peptídeo natriurético NT-proBNP junto com o avanço da DVCM em cães da raça Poodle (Tab. 2 e Fig. 1).

Tabela 2. Mediana, intervalo interquartil (IQ), valores mínimos e máximos da concentração plasmática do NT-proBNP (pmol/L) dos cães Poodles, atribuídos a diferentes estágios da DVCM ACVIM (2009)

\begin{tabular}{cccccc}
\hline & Estágios & $\begin{array}{c}\text { Mediana } \\
(\mathrm{pmol} / \mathrm{L})\end{array}$ & Intervalo IQ & $\begin{array}{c}\text { Mín. } \\
(\mathrm{pmol} / \mathrm{L})\end{array}$ & $\begin{array}{c}\text { Máx. } \\
(\mathrm{pmol} / \mathrm{L})\end{array}$ \\
\cline { 2 - 6 } NT-proBNP & $\mathrm{A}$ & $551^{\mathrm{a}}$ & $523,5 / 600,25$ & 250 & 661 \\
& $\mathrm{~B} 1$ & $302^{\mathrm{a}}$ & $261 / 523$ & 250 & 709 \\
& $\mathrm{~B} 2$ & $1.033^{\mathrm{b}}$ & $544,75 / 1.325,25$ & 328 & 2.096 \\
& $\mathrm{C}$ & $954^{\mathrm{b}}$ & $618,25 / 2.554,75$ & 394 & 3.178 \\
& $\mathrm{D}$ & $5.541^{\mathrm{c}}$ & $3.086,25 / 5.991,25$ & 2.265 & 8.872 \\
\hline
\end{tabular}

Nos valores seguidos por diferentes letras (“a”, “b”, “c”), não houve alterações estatísticas significativas (P>0,05). 
NT-proBNP

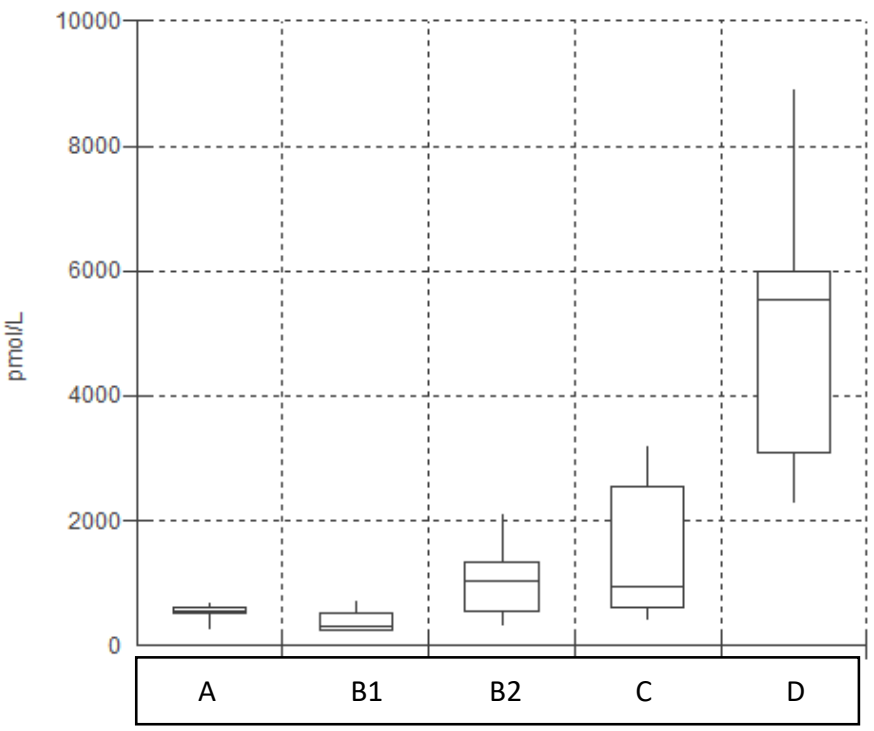

Figura 1. Concentração plasmática do NT-proBNP (pmol/L) de todos os cães do estudo, de acordo com os estágios da DVCM (ACVIM, 2009). As linhas centrais dentro de cada bloco indicam a mediana do grupo; as linhas superiores, o primeiro quartil; e as linhas inferiores dos blocos indicam o terceiro quartil. Linhas verticais externas aos blocos são valores máximos e mínimos.

Após análise da curva ROC, houve determinação do ideal cut-off (>709pmol/L), para discriminar os cães verdadeiros positivos (aumento cardíaco) dos verdadeiros negativos (ausência de aumento cardíaco). O teste do biomarcador cardíaco NTproBNP obteve a sensibilidade de $75 \%$ e a especificidade de $100 \%$. A área sob a curva (AUC) foi de 0.87 (Fig. 2).

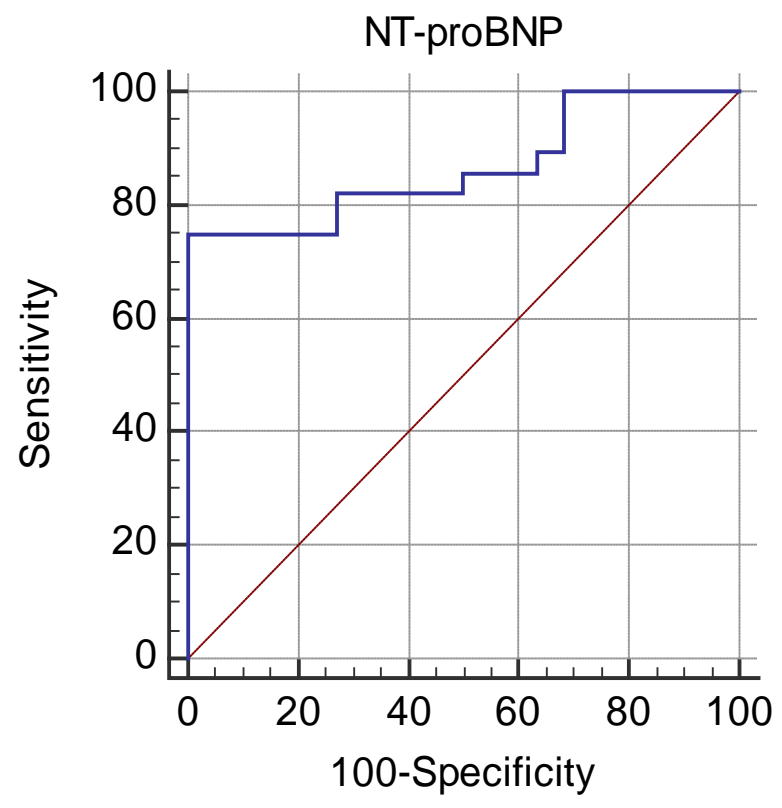

Figura 2. Curva ROC representando a sensibilidade de $75 \%$ e a especificidade de $100 \%$ do teste NTproBNP em cães da raça Poodle com DVC. Área sob a curva (AUC) de 0.87. 
Não houve diferença significativa entre as variáveis dos exames laboratoriais $(\mathrm{P}>0,05)$ em relação ao grupo controle. A urinálise foi utilizada como fonte simples complementar para análise de distúrbios do trato urinário, combinada com a avaliação bioquímica, em geral sem variações significantes $(\mathrm{P}>0,05)$ entre todos os grupos e o grupo controle (Tab. 3). Os resultados dos exames cardiológicos estão apresentados na Tab. 4.

Tabela 3. Médias e desvios-padrão referentes aos parâmetros hematológicos, bioquímicos séricos e urinários dos cães Poodles separados de acordo com as diretrizes para doença valvular crônica do ACVIM

\begin{tabular}{ccccccc}
\hline & $\mathrm{A}$ & $\mathrm{B} 1$ & $\mathrm{~B} 2$ & $\mathrm{C}$ & $\mathrm{D}$ & Referência \\
\hline VG (\%) & $47 \pm 5$ & $48 \pm 5$ & $45 \pm 6$ & $46 \pm 7$ & $44 \pm 6$ & $37-55 \%$ \\
LG (x10 $/ \mu \mathrm{L})$ & $10.2 \pm 2.2$ & $9.9 \pm 1.9$ & $9.0 \pm 2.7$ & $10.4 \pm 3.5$ & $15.7 \pm 9.1$ & $6.0-17.0$ \\
Ureia (mg/dL) & $37 \pm 11$ & $40 \pm 15$ & $43 \pm 12$ & $53 \pm 33$ & $117 \pm 80$ & $21-60$ \\
Creatinina (mg/dL) & $0,8 \pm 0,2$ & $0,8 \pm 0,2$ & $0,8 \pm 0,3$ & $0,8 \pm 0,2$ & $1,1 \pm 0,8$ & $0.5-1.5$ \\
AST (UI/L) & $41 \pm 10$ & $43 \pm 15$ & $42 \pm 12$ & $51 \pm 18$ & $71 \pm 40$ & $23-66$ \\
ALT (UI/L) & $59 \pm 11$ & $81 \pm 23$ & $64 \pm 13$ & $80 \pm 19$ & $196 \pm 41$ & $21-102$ \\
FA (UI/L) & $46 \pm 24$ & $58 \pm 23$ & $51 \pm 21$ & $48 \pm 32$ & $88 \pm 73$ & $20-156$ \\
Ptn totais (g/dL) & $6.8 \pm 0.2$ & $6.7 \pm 0.4$ & $6.5 \pm 0.7$ & $7.7 \pm 1.2$ & $7.3 \pm 1.2$ & $5.4-7.1$ \\
Rl: Alb/ptn & $0.7 \pm 0.1$ & $0,7 \pm 0.1$ & $0.8 \pm 0.2$ & $0.6 \pm 0.2$ & $0.7 \pm 0.1$ & $0.59-1.1$ \\
Ptn plasm (g/dL) & $7.4 \pm 0.6$ & $7.4 \pm 0.7$ & $7.2 \pm 0.8$ & $7.5 \pm 0.7$ & $7.8 \pm 0.8$ & $6-8$ \\
pH urinário & $6 \pm 0$ & $6 \pm 0.3$ & $6 \pm 0.5$ & $6 \pm 0.6$ & $5 \pm 0.6$ & $5,0-6,5$ \\
Densidade urinária & 1.016 & 1.018 & 1.020 & 1.020 & 1.020 & $1.015-1.045$ \\
\hline
\end{tabular}

VG volume globular; LG leucometria global; AST aspartato transaminase; ALT alanina transaminase; FA fosfatase alcalina; PTN TOTAIS proteínas totais; RL:Alb/ptn relação albumina/proteína; PTN PLASM proteína plasmática.

Tabela 4. Média, desvio-padrão, valores mínimos e máximos dos parâmetros clínico- cardiológicos dos cães Poodles, separados pelos estágios da doença valvular crônica de acordo com as diretrizes do ACVIM

\begin{tabular}{|c|c|c|c|c|c|c|}
\hline & $\mathrm{A}$ & B1 & $\mathrm{B} 2$ & $\mathrm{C}$ & $\mathrm{D}$ & Referência \\
\hline PAS (mmHg) & $\begin{array}{c}147 \pm 28 \\
(103-181)\end{array}$ & $\begin{array}{c}142 \pm 25 \\
(109-183)\end{array}$ & $\begin{array}{c}146 \pm 35 \\
(86-194)\end{array}$ & $\begin{array}{c}150 \pm 34 \\
(109-206)\end{array}$ & $\begin{array}{c}167 \pm 34 \\
(97-206)\end{array}$ & 137 \\
\hline PAD (mmHg) & $\begin{array}{c}95 \pm 22 \\
(45-127)\end{array}$ & $\begin{array}{c}95 \pm 15 \\
(79-134)\end{array}$ & $\begin{array}{c}113 \pm 40 \\
(57-182)\end{array}$ & $\begin{array}{c}99 \pm 20 \\
(63-131)\end{array}$ & $\begin{array}{c}109 \pm 15 \\
(83-131)\end{array}$ & 82 \\
\hline PAM (mmHg) & $\begin{array}{c}113 \pm 27 \\
(82-168)\end{array}$ & $\begin{array}{c}107 \pm 20 \\
(88-149)\end{array}$ & $\begin{array}{c}116 \pm 34 \\
(65-178)\end{array}$ & $\begin{array}{c}119 \pm 26 \\
(76-157)\end{array}$ & $\begin{array}{c}126 \pm 25 \\
(87-157)\end{array}$ & 102 \\
\hline $\begin{array}{l}\text { Freq. Cardíaca } \\
\text { (bpm) }\end{array}$ & $\begin{array}{c}133 \pm 15 \\
(120-160)\end{array}$ & $\begin{array}{c}124 \pm 24 \\
(100-160)\end{array}$ & $\begin{array}{c}124 \pm 16 \\
(100-160)\end{array}$ & $\begin{array}{c}144 \pm 24 \\
(100-180)\end{array}$ & $\begin{array}{c}147 \pm 32 \\
(100-200)\end{array}$ & $60-180$ \\
\hline AE:Ao & $\begin{array}{c}1,1 \pm 0.1 \\
(1,0-1,5)\end{array}$ & $\begin{array}{c}1 \pm 0.1 \\
(0,8-1,2)\end{array}$ & $\begin{array}{c}1,7 \pm 0.3 \\
(1,1-2.5)\end{array}$ & $\begin{array}{c}1,7 \pm 0.1 \\
(1,5-2,0)\end{array}$ & $\begin{array}{c}1,9 \pm 0.3 \\
(1,6-2,8)\end{array}$ & $0,8-1,2$ \\
\hline DVE-d (mm) & $\begin{array}{l}21.9 \pm 2.8 \\
(17-26)\end{array}$ & $\begin{array}{l}20.3 \pm 3.1 \\
(13-45)\end{array}$ & $\begin{array}{l}20.7 \pm 4.6 \\
(12-26)\end{array}$ & $\begin{array}{l}26.3 \pm 3.8 \\
(21-32)\end{array}$ & $\begin{array}{c}29.2 \pm 10.1 \\
(15-25)\end{array}$ & $20.6-22,7$ \\
\hline DVE-s (mm) & $\begin{array}{c}12.1 \pm 2.5 \\
(5-14)\end{array}$ & $\begin{array}{c}12.7 \pm 3.5 \\
(9-20)\end{array}$ & $\begin{array}{c}14.8 \pm 4.0 \\
(8-21)\end{array}$ & $\begin{array}{l}16.5 \pm 2.5 \\
(10-19)\end{array}$ & $\begin{array}{c}17.8 \pm 7.6 \\
(7-31)\end{array}$ & $11,7-13,6$ \\
\hline FEC (\%) & $\begin{array}{c}39 \pm 3.3 \\
(34-44)\end{array}$ & $\begin{array}{c}37 \pm 4.4 \\
(32-46)\end{array}$ & $\begin{array}{c}39 \pm 3.7 \\
(32-44)\end{array}$ & $\begin{array}{l}44 \pm 11.6 \\
(30-59)\end{array}$ & $\begin{array}{c}39 \pm 8.9 \\
(20-59)\end{array}$ & $28-44$ \\
\hline $\mathrm{E}: \mathrm{A}$ & $\begin{array}{c}1,5 \pm 0.2 \\
(1,1-1,9)\end{array}$ & $\begin{array}{c}1,5 \pm 0.3 \\
(0,8-1,9)\end{array}$ & $\begin{array}{c}1,5 \pm 0.4 \\
(1,2-4,6)\end{array}$ & $\begin{array}{c}1,5 \pm 0.3 \\
(1,0-2,0)\end{array}$ & $\begin{array}{c}2 \pm 1.1 \\
(0,8-2,3)\end{array}$ & $1,9-2,7$ \\
\hline
\end{tabular}

PAS pressão arterial sistólica; PAD pressão arterial diastólica; PAM pressão arterial média; mmHg unidade milímetro mercúrio; bpm batimentos por minutos; AE:Ao relação átrio esquerdo/aorta; DVE-d diâmetro do ventrículo esquerdo em diástole; DVE-s diâmetro do ventrículo esquerdo em sístole; FEC fração de encurtamento; E:A relação onda E/onda A. Fonte dos valores de referências (BOON, 2011).

\section{DISCUSSÃO}

O estudo em questão foi realizado com o objetivo de mensurar o biomarcardor cardíaco NT-proBNP somente em cães da raça Poodle com DVCM, classificados segundo as diretrizes do ACVIM (2009) para o diagnóstico e o tratamento da DVC, e em um grupo controle. Portanto, acredita-se que os resultados apresentados representem um avanço nos estudos para utilização de novas ferramentas para um diagnóstico principalmente precoce e eficiente da referida doença na clínica de pequenos animais. 
De modo similar a estudos anteriores (MacDonald et al., 2003; Chetboul et al., 2009; Oyama et al., 2009; Ebisawa et al., 2012; Wolf et al., 2012; Kanno et al., 2015), o presente estudo evidenciou aumento significativo nas concentrações plasmáticas do NT-proBNP, em conjunto com o avanço dos sintomas e dos sinais clínicos da DVCM. Entretanto, este estudo não evidenciou relação entre os valores plasmáticos de NT-proBNP e os critérios peso e sexo, tal qual relatado por Misbach et al. (2013) e Sjostrand et al. (2014). Conforme observado por Hezzell et al. (2012), os animais mais idosos apresentaram maior risco de insuficiência cardíaca. No presente estudo, foi igualmente constatado que a idade dos cães avançou acompanhando a evolução da DVCM de acordo com os estágios determinados pelas diretrizes do ACVIM (2009) para essa enfermidade.

Anterior ao presente estudo, Couto et al. (2015) relatou, em cães Greyhound saudáveis, retirados das corridas, um valor médio do NT-proBNP de 945pmol/L (512 - 2127pmol/L). Hezzell et al. (2012), entretanto, em seu estudo, chegaram a utilizar dois Poodles saudáveis misturados a outras raças (n=30 cães) e obtiveram média de 324pmol/L (167 - 530pmol/L). Os valores plasmáticos do NT-proBNP obtidos no grupo controle do presente estudo (estágio A; ACVIM, 2009), em que foram utilizados 12 Poodles saudáveis, podem colaborar futuramente para comparações entre as próximas pesquisas com a raça Poodle. A análise estatística da curva ROC obteve um valor ideal de cut-off (>709pmol/L), com $75 \%$ de sensibilidade e $100 \%$ de especificidade, em que valores acima do valor ideal de corte já indicavam aumento cardíaco e estresse miocárdico comprovados pelos exames ecocardiográficos. Para os cães do estágio B1, não houve diferença $(\mathrm{P}>0,05)$ quando comparados aos animais do grupo controle. A mensuração dos níveis plasmáticos do NTproBNP nestes pacientes assintomáticos e sem alterações hemodinâmicas, no presente estudo, como afirmaram Sharma et al. (2015), mostrouse excelente auxiliar no diagnóstico e forneceu resultados valiosos para discriminar os riscos causados pela DVCM.

Os valores abaixo de 900pmol/L dos cães $(n=4)$ do grupo B2 podem ser explicados pela degradação da amostra no tubo armazenado sem inibidor de proteases, o que levou à diminuição no resultado do biomarcador (Hezzell et al., 2012). Além disso, em razão da influência das diversas conformações corpóreas existentes nos cães, a variabilidade das medidas ecocardiográficas ocorre não somente entre as espécies animais, mas também entre as raças caninas (Yamato et al., 2006). Por outro lado, cães do grupo B2 com níveis plasmáticos de 90L até 1.500pmol/L são cães $(\mathrm{n}=3)$ com a DVCM, porém sem sinais clínicos, sem chamar atenção para a existência de estresse miocárdico e o aumento cardíaco, o que indicou precocemente a necessidade de acompanhamento clínico cardiológico. Os cães ( $n=2$ ) desse grupo B2 em que os níveis plasmáticos foram acima de $1.501 \mathrm{pmol} / \mathrm{L}$ foram considerados cães com futuro risco de desenvolverem ICC e sintomas clínicos graves da DVCM de forma aguda e até mesmo falha cardíaca (Wolf et al., 2013).

No grupo C, alguns cães $(n=5)$ portadores da DVCM, com sintomas discretos a moderados da ICC e com valores do NT-proBNP baixos $(<900 \mathrm{pmol} / \mathrm{L})$, representaram a triagem que esse biomarcador cardíaco faz entre distúrbios crônicos do trato respiratório concomitantes com a DVCM (Oyama et al., 2009); também pode ser discutida a degradação da amostra no armazenamento do biomarcador. Um cão $(n=1)$ desse grupo apresentou valor entre 90L e 1500pmol/L com DVCM e sintomatologia clínica significativa; esse paciente respondeu positivamente ao tratamento padrão (Wolf et al., 2012). Já os cães desse grupo C com níveis plasmáticos acima de 1800pmol/L $\quad(n=4)$ correspondem a animais hospitalizados com sintomas clínicos graves da ICC (ex.: síncopes e angústias respiratórias), causados pela DVCM com chance de falha cardíaca e necessidade do tratamento terapêutico intensivo (Takemura et al., 2009).

Os cães do estágio D foram refratários ao tratamento padrão da DVCM com sintomas avançados da ICC e alguns com necessidades urgentes de cuidados hospitalares. Neste estudo, todos os animais desse grupo apresentaram níveis plasmáticos do NT-proBNP muito acima de $1800 \mathrm{pmol} / \mathrm{L}$, o que significa um alto estiramento e estresse cardíaco, que ocasionaram falência miocárdica (MacDonald et al., 2003). O presente estudo utilizou os resultados básicos dos testes laboratoriais para ajudar a identificar os cães com a ICC descompensada e, assim, prestar 
melhor assistência a esses cães hospitalizados, a fim de minimizar o risco de morte hospitalar (Ostrowska et al., 2016).

Este estudo encontrou limitações devido à falta de tubos com inibidores de proteases para conservação das amostras. Isso pode ter favorecido a degradação do biomarcador NTproBNP antes do envio para a análise laboratorial, sendo, desse modo, explicados os resultados com valores abaixo do esperado. Porém, com o auxílio diagnóstico da ecocardiografia e de exames clínicos complementares, as concentrações plasmáticas do biomarcador foram consideradas falsonegativas para aumento cardíaco. Vale ressaltar que os valores de referência para o NT-proBNP possuem variabilidades individuais de acordo com o tipo de teste aplicado, a especificidade e o manuseio das amostras, que são fatores que devem ser considerados quando se faz comparação com resultados de outros estudos.

\section{CONCLUSÃO}

Os níveis plasmáticos do peptídeo natriurético NT-proBNP em cães da raça Poodle aumentam com a evolução da DVCM, sendo os estágios B2, C e D (ACVIM, 2009) aqueles em que se observam os maiores valores desse biomarcador. Além disso, utilizando-se o valor de corte de $>709 \mathrm{pmol} / \mathrm{L}$, foi possível identificar o remodelamento cardíaco com sensibilidade de $75 \%$ e especificidade de $100 \%$. Para os cães do estágio B2 (ACVIM, 2009), a mensuração do biomarcador cardíaco NT-proBNP mostrou ser uma ótima ferramenta para diagnosticar, de forma precoce, o aumento cardíaco antes da sintomatologia clínica da DVCM.

\section{BIBLIOGRAFIA}

AYRES, M.; AYRES, J.R.M.; AYRES, D.A.; SANTOS, A.S. BioEstat 2.0 aplicações estatísticas nas áreas das ciências biológicas e médicas. Brasília: CNPq, 2000. 259p.

BARRETTO, A.C.P.; CARLO, C.H.; CARDOSO, J.N. et al. Papel dos níveis de BNP no prognóstico da insuficiência cardíaca avançada descompensada. Arq. Bras. Cardiol., v.100, p.281-287, 2013.
BOON, J.A. Veterinary echocardiography. 2.ed. Ames: Wiley-Blackwell, 2011. p.538-541.

BORGARELLI, M.; HAGGSTROM, J. Canine degenerative myxomatous mitral valve disease: natural history, clinical presentation and therapy. Vet. Clin. Small Anim., v.40, p.651-663, 2010.

CHETBOUL, V.; SERRES, F.; TISSIER, R. et al. Association of plasma N-terminal Pro-B-type natriuretic peptide concentration with mitral regurgitation severity and outcome in dogs with asymptomatic degenerative mitral valve disease. J. Vet. Intern. Med., v.16, p.984-994, 2009.

CÔTÉ, E.; EDWARDS, J.N.; ETTINGER, S.J. et al. Management of incidentally detected heart murmurs in dogs and cats. J. A. Vet. Med. Assoc., v.246, p.1076-1088, 2015.

COUTO, K.M. et al. PlasmaN-terminalpro-Btypenatriureticpeptide concentrationin healthy retired racingGreyhounds. Vet. Clin. Pathol., v.44-43, p.405-409, 2015.

DETWEILER, D.K.; PATTERSON, D.F. The prevalence and types of cardiovascular disease in dogs. Ann. N.Y. Acad. Sci., v.127, p.481-516, 1965.

EBISAWA, T.; OHTA, Y.; FUNAYAMA, M.; MORITA, K.; UECHI, M. Clinical use of Nterminal pro-brain natriuretic peptide concentrations for assessing the severity and prognosis of myxomatous mitral valve disease in dogs. Intern. J. Appl. Res. Vet. Med., v.10, p.234242, 2012.

FOX, P.R.; SISSON, D.; MOISE, N.S. Textbook of canine and feline cardiology - principles and clinical practice. 2.ed. Philadelphia: Saunders, 1999. p.41-171.

GAGGIN, H.K.; JANUZZI, Jr. J.L. Biomarkers and diagnostics in heart failure. Biochim. Biophys. Acta., v.1832, p.2442-2450, 2013.

HEZZELL, M.J.; BOSWOOD, A.; CHANG, Y.M. et al. The combined prognostic potential of serum high-sensitivity cardiac troponin I and Nterminal pro-B-type natriuretic peptide concentrations in dogs with degenerative mitral valve disease. J. Vet. Intern. Med., v.26, p.302311, 2012. 
KANNO et al. Plasma atrial natriuretic peptide and $\mathrm{N}$-terminal pro B-type natriuretic peptide concentrations in dogs with right-sided congestive heart failure. J. Vet. Med. Sci., v.78, p.535-542, 2015.

MacDONALD, K.A.; KITTLESON, M.D.; MUNRO, C.; KASS, P. Brain natriuretic peptide concentration in dogs with heart disease and congestive heart failure. J. Vet. Intern. Med., v.17, p.172-177, 2003.

MISBACH, C.; CHEBOUL, V.; CONCORDET, D. et al. Basal plasma concentrations of $\mathrm{N}$ terminal pro-B-type natriuretic peptide in clinically healthy adult smack size dogs: effect of body weight, age, gender and breed, and reference intervals. Res. Vet. Sci., v.95, p.879885, 2013.

OSTROWSKA, M.; OSTROWSKI, A.; LUCZAK, M. et al. Basic laboratory parameters as predictors of in-hospital death in patients with acute decompensated heart failure - data from a large single-centre cohort. Pol. Heart J., v.75, p.157-163, 2016.

OYAMA, M.A. et al. Assessment of serum Nterminal pro-B-type natriuretic peptide concentration for differentiation of congestive heart failure from primary respiratory tract disease as the cause of respiratory signs in dogs. J. Am. Vet. Med. Assoc., v.235, p.1319-1325, 2009.

OYAMA, M.A. The use of NT-proBNP assay in the management of canine patients with heart disease. Vet. Clin. N. Am. Small Anim. Pract., v.40, p.545-458, 2010.

PANT, S.; DESHMUKH, A.; NEUPANE, P.; KUMAR, K.M.B; VIJASHANKAR, C.S. Cardiac biomarkers. Novel strategies in ischemic heart disease. [s.l.]: [s.n.], 2012. p.1742.

PERNOMIAN, L. Efeitos vasculares induzidos pelo peptídeo natriurético tipo C (CNP). 2011. 147f. Dissertação (Mestrado em Farmacologia) Universidade de Medicina de Riberão Preto, São Paulo, SP.
REAGAN W.J.; ROVIRA A.R.I.; DeNICOLA D.B. Atlas de hematologia veterinária. 2.ed. [Rio de Janeiro]: Revinter, 2011. 120p.

SHARMA, A.; AHMED, V.; GARG, A.; AGGARWAL, C. Clinical applications of natriuretic peptides in assessment of valvular heart disease. [Cairo]: Hindawi Publishing Corporation, 2015. p.1-6.

SIMONS, J.E.; WAUCHOPE, A.C.D. Evaluation of natriretic peptide recommendations in heart failure clinical practice guidelines. Clin. Biochem., v.49, p.1-8, 2015.

SJOSTRAND, K. et al. Breed differences in natriuretic peptides in healthy dogs. J. Vet. Intern. Med., v.28, p.451-457, 2014.

TAKEMURA, N. et. al. Evaluation of plasma Nterminal pro-brain natriuretic peptide (NTproBNP) concentrations in dogs with mitral valve insufficiency. J. Vet. Med. Sci., v.71, p.925-929, 2009.

WOLF, J.; GERLACH, N.; WEBWR, K.; KLIMA, A.; WESS, G. Lowered N-terminal proB-type natriuretic peptide levels in response to treatment predict survival in dogs with symptomatic mitral valve disease. J. Vet. Cardiol., v.14, p.399-408, 2012.

WOLF, J.; GERLACH, N.; WEBWR, K.; KLIMA, A.; WESS, G. The diagnostic relevance of NT-proBNP and proANP 31-67 measurements in staging of myxomatous mitral valve disease in dogs. Vet. Clin. Pathol., v.42, p.196-206, 2013.

YAMATO, R.J.; LARSON, M.H.M.A.; MIRANDOLA, R.M.S. et al. Parâmetros ecocardiográficos em modo unidimensional de cães da raça Poodle miniatura, clinicamente sadios. Ciênc. Rural, v.36, p.142-148, 2006.

YONEZAWA, L.A.; SILVEIRA V.F.; MACHADO L.P.; KOHAYAGAWA, A. Marcadores cardíacos na Medicina Veterinária. Ciênc. Rural, v.40, 9p., 2010. 